\title{
Research of embedded remote monitor and control network based on Internet technology
}

\author{
Haoran Song \\ Marine college of Shandong jiaotong university, Weihai, Shandong,China
}

Keywords: Embedded, remote monitoring network; CAN bus; Linux and real-time

\begin{abstract}
Remote monitoring refers to the local computer with the Web as a platform for communication to remote computers or devices to monitor and control. This project is combining the Internet and embedded devices, uses the local computer through the Internet to embedded devices and remote slave monitor. Embedded device with small size, real-time performance, high reliability, functional characteristics such as design and implementation of a complete network monitoring system. The system with step motor controlled, system-on-chip AT89552 stepper motor controller chip, through CAN communication with site links to embedded systems, PC access by Intemet embedded systems, monitoring and control of the control.
\end{abstract}

\section{Introduction}

Remote monitoring refers to the local computer through the network system on the remote computer or device to monitor and control. In recent years, the rapid development of the Internet, achieving a wide range of high-speed transmission of information and resource sharing, greatly facilitates our lives. Embedded device with small size, real-time performance, high reliability and functional characteristics. Internet and embedded systems is an inevitable trend.

With the rapid development of information technology, causing the transformation of automation system, gradually forming the basis of network integration automation of enterprise information system, field bus is the new technology developed to conform to the change. Fieldbus at bottom of production equipment can be connected together to form controls at the bottom of the network, realizing the enterprise information integration and automation.

RS-232 bus of communications distance short, only achieved point-to-point communications, cannot directly achieved more points communications, and early of some bottom site control equipment often are has RS-232 interface and not has CAN interface, cannot directly connection to CAN bus network Shang, to achieved original resources of repeat using, save networking cost, this design in the development has a will RS 12 made bus conversion to CAN bus of agreement conversion device. The protocol converter to RS 12 per cent has direct access to the CAN bus interface device on the network.

These technologies can be applied to industrial control systems and equipment, is conducive to maintaining synchronization with a mainstream communications technology development of industrial control network, so that it has a very strong capacity for sustainable development. Therefore, research on these technologies, have a certain significance.

\section{Embedded remote monitoring network system design}

Network monitoring system system consists of three main components, namely PC, embedded controllers, system-on-chip controller. Through Internet communication and CAN communicate two ways to connect the three main constitutes a Internet as the transmission medium, embedded systems and system-on-chip controller, computer monitor on PC, with stepping motor for control of remote monitoring systems. In the system design process requires the use of embedded systems, embedded Internet access method, CAN bus, in these technologies based on the embedded remote monitoring network frame based on the Internet. 
Embedded systems are State of the art computer technology, semiconductor technology, electronic technology and product of combining various industry specific applications, it has decided it must be a technology-intensive, capital-intensive, highly decentralized and integrated system of innovative knowledge.

Including embedded software, embedded system and embedded hardware in two parts. Hardware support, software is soul. All embedded systems require software to provide variety, and using special functions. Design of an embedded system, it is necessary to find a suitable software and hardware platforms.

TCP/IP protocol which can be used in a variety of networks, can be on different physical networks, topology shielding, can afford to provide a common, unified network services. From the user point of view, TCP/IP protocol suite is a unified whole, it is independent of specific physical networks, provide a common network services to users. In the TCP/IP Protocol, the receiving process is a process of unpacking, always from the lower receiver function call in the module in the upper module of the receiver function; sending process is a continuous packaging process, always above the send function call in the module below the send function module.

CAN-bus is a multi-master bus, communication medium can be twisted, coaxial cable or optical fiber. CAN protocol is one of the biggest feature of the abolition of the traditional station address coding used to encode communication block. The advantage of using this method can in theory unlimited number of nodes in the network. CAN-bus is the main competition of bus structure, with multiple main station and scattered broadcast serial bus arbitration and communication features.

CAN bus has communications rate make, easy achieved, and price high many features, is a has formed international standards of site bus, this is CAN bus application Yu many field, has strong often competitiveness of important reasons; in future a time within, CAN bus by has of high reliability, real-time sex and flexibility, highlight advantages, plus widely of application based, CAN bus will increasingly by welcomes.

The system consists of three components: monitoring, field master end and field end. Scene between master and master through Internet connections, master control via CAN bus connection to the field side.

Site master Tip: hardware design including power circuit, reset circuit, memory circuits, such as software under Linux platform is designed with Internet and two-way send and receive capability CAN software. The master side connection of both Internet and CAN communicate, is the link the entire system.

Field end: perform end design and implement based on CAN communication of stepper motor control with rotary encoder systems, the system CAN run communications receives control commands and sends the status, including hardware and software design. Hardware design using AT89552 microcontroller, rotary encoders to realization of MCU and photoelectric coupling apparatus for stepper motor control software design through the timer interrupts and comparative interrupted, CAN receive function CAN send function and stepper motor control functions.

Protocol Design: the protocol converter for CAN communication protocol and RS 12 per cent and protocol conversion, implementation does not have a CAN bus interface equipment can be directly attached to the bus network. AT89552 single-chip SJA1000 and PCA82C250 constitutes hardware; software CAN interrupt reception, CAN send serial interrupt receives and sends serial child functions.

\section{Implementation of embedded WEB server}

WEB server as user programs on the master side of the system, accept the monitoring of requests, gathered the field control end of run information, sends it to the browser, on the other hand requested by the monitor send control information to the monitoring equipment at the scene.

System of hardware part design provides has network interface, to improve bear of network access capacity and operation sex, in system in the design achieved has embedded WEB server. because is embedded equipment, so server of capacity, run speed, consistency and function support these 
aspects are need consider. traditional of WEB server has is high of operation processing capacity and is big of storage space, in its Shang run of most is is responsible for of server program, And traditional WEB servers is not based on real time operating system as the software platform, so the traditional WEB technical solutions cannot be directly used to construct the WEB server in industrial control systems, this configuration is 16 or 32-bit embedded systems cannot be achieved.

In embedded Linux operating system in the, main of Web server has three species: httfd, Thttpd, BOA. which httPd only support static page, apparently not for senior application, THTTPD and BOA by provides of function basic as, but THTTPD in run process in the by need of resources to far is greater than BOA. so this system in the selection Boa and CGI, then according to need for crop, building out for this system of embedded WEB server.

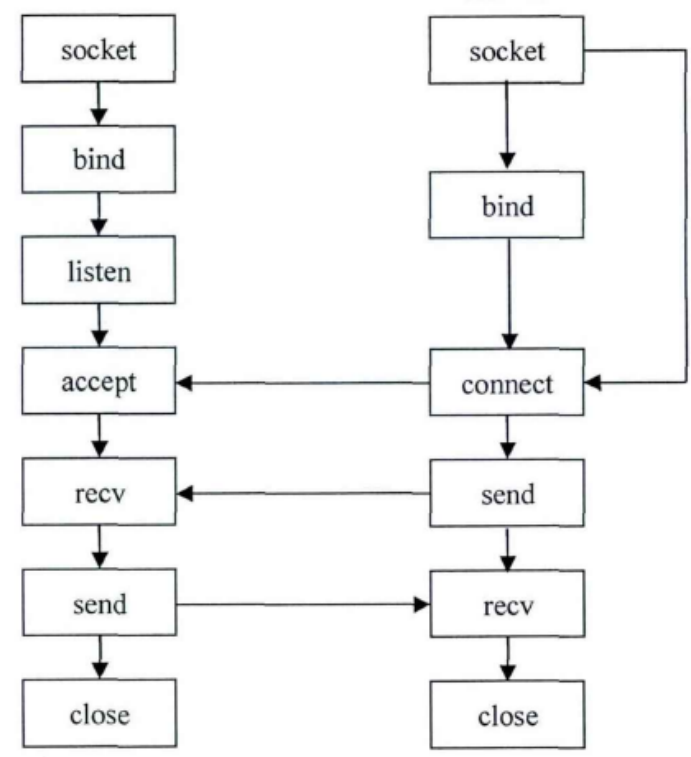

Fig . 1 Use TCP Soeket programming

To achieved user and Web server of interactive, General used table single as CGI of entered interface. table alone browser to Web server submitted information of tool, table alone user in page in the fill in information of form, its role is accept user information and will its submitted to Web server Shang specific of program for processing. table single contains has allows user and select information of table single domain. Dang customer in page of table single domain in the fill in information and press Hou, table single content on was sent to Web server Shang; Web server and CGI program of interactive process is through environment variable to achieved of. environment variable is by some Web server software application of, save user information of memory district composition of. once Web server software began run, these environment variable on exists Yu Web server in the. through environment variable, Web server can collection on server of himself detailed information, user information and user requests information three a aspects of content.

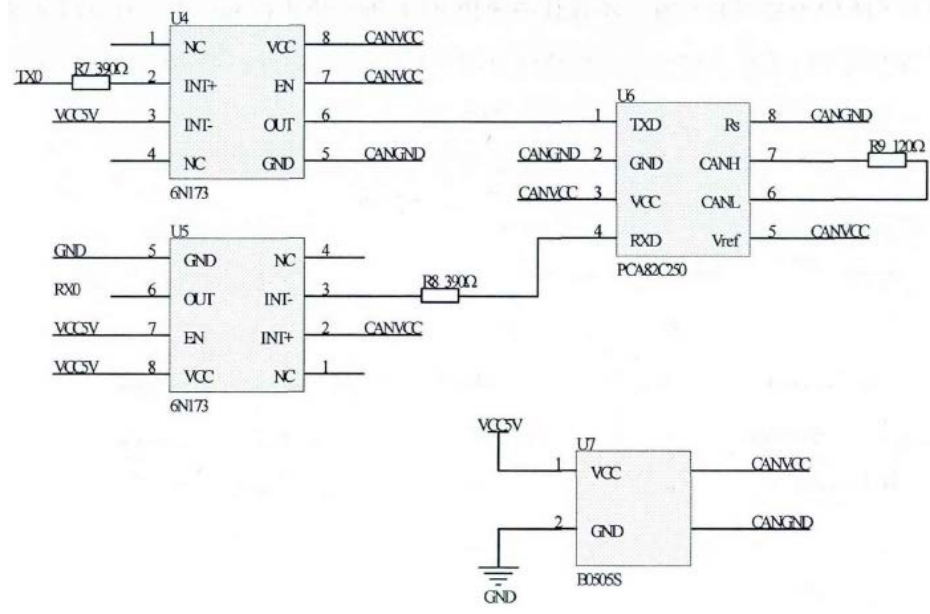

Fig . 2 CAN bus interface circuit 


\section{Research of CAN bus in real time}

Real time is an important factor in the industrial control field. CAN bus with multiple primary sent! deterministic priority arbitration mechanism of CAN-bus communication reliability is guaranteed! timeliness and flexibility. in the process of communication, if there are collisions, lower priority sites will be active exit, high-priority node not affected continue to transmit data, which greatly saves bus arbitration practice, To guarantee has data of real-time sex. but this static priority level mechanism of a shortcomings is cannot equal of for high priority level and low priority level site distribution bandwidth, in network load is big Shi, low priority level site will in times competition bus right Shi failed, to led to low priority level site transmission produced uncertainty of delay, even cannot normal sent. in this section in the main for bottom of CAN bus network of real-time sex for analysis.

FIFO memory for 64-byte, bit-stream processor is used CAN protocol frames received! President and handle error instructions, and so on. SJA100O, first enter the FIFO memory for new messages, and only enter this message receive buffer can be CPU, thus Ao Zheng CPU processing to receive new messages as well as messages.

Dynamic priority algorithm is an algorithm that dynamically changing sites priority over time, compared with the original static-priority algorithm, this algorithm allows equal access to competing bus per site, that is, to equal distribution of bandwidth to ensure message transmission in real time.

Initial situation Xia all site are has prior distribution good of priority level, in not occurred conflict of situation Xia, by original priority level to completed data of transfer. Dang occurred conflict zhihou, high priority level site can smooth completed data sent, to can let competition failed of site in next competition in the success of probability variable big, can will competition failed of site improve a priority grade participate in next competition, even failed has several times, then the site of priority level also is high has, competition wins of probability will is big, But note that are lower priority site in raising the priority of access right to use bus and after the successful completion of the send data, priority needs to be reduced to the initial priority, in order to ensure the normal operation of the network.

Through experiment data can see, with load rate of increased standard CAN scheduling Xia of average delay time almost into index increased, and dynamic priority level scheduling Xia of average delay time increased rarely. dynamic priority level dynamic priority level scheduling algorithm relative Yu standard CAN scheduling algorithm, in network real-time sex aspects has has must of improve, with network load rate of increased, low priority level node get bus right of chances increased.

\section{References}

[1]Wang Tianmiao. embedded systems design and development. Beijing: Tsinghua University Press, 2004.

[2]Martin Wojcik, Ganesh Ranganathan. Ethemet and the web gang up Proeess monitoring and control ISA:InTech,2009.9:21-32

[3]Du chunlei. ARM architecture and programming [m]. Beijing: Tsinghua University Press, 2009

[4]Ma Zhongmei, and so on. ARM\&Linux embedded systems tutorial [m]. Beijing: Beijing University of Aeronautics and Astronautics press, 2008

[5]Tian Ze. embedded system development and application [m], Beijing: Beijing University of Aeronautics and Astronautics press, 2005

[6]Gu Yan. based on data warehouse technology in hospital information systems (HIS) Scheme [j]. computer systems applications. 2005

[7]Yi Jing. and implementation of data mining technology in the hospital information [j]. Chongqing University of medical sciences 2007.

[8]Kantardzic M. Data Mining Concept、Models、Methods and Algorithms[M]. IEEE Press.2002 